\title{
MOTIVAÇÃO DE JOVENS E ADULTOS PARA O TREINAMENTO DO ATLETISMO
}

\author{
Renato Siqueira de Souza \\ Universidade Federal de Juíz de Fora, Juiz de Fora, Minas Gerais, Brasil \\ Jorge Roberto Perrout de Lima \\ Universidade Federal de Juíz de Fora, Juiz de Fora, Minas Gerais, Brasil \\ Francisco Zacaron Werneck \\ Universidade Federal de Ouro Preto, Ouro Preto, Minas Gerais, Brasil \\ Maurício Gattás Bara Filho \\ Universidade Federal de Juíz de Fora, Juiz de Fora, Minas Gerais, Brasil \\ Jefferson Verbena de Freitas \\ Universidade Federal de Juíz de Fora, Juiz de Fora, Minas Gerais, Brasil
}

\begin{abstract}
Resumo
O estudo investigou diferenças na motivação de atletas do atletismo, comparando grupos de provas, nível competitivo, categoria e sexo. Avaliamos a motivação através do questionário (SMS-BR28) de 133 atletas, 56\% do sexo masculino e $44 \%$ do sexo feminino. Não teve diferenças significativas entre grupos de provas e nível competitivo, nível de significância $(\mathrm{P}<0,05)$. Houve diferença significativa comparando à motivação de juvenis e adultos vs pré-mirim e mirim. Concluímos que a motivação para treinar Atletismo independe da prova disputada, atletas juvenis, sub-23 e adultos são mais motivados intrinsecamente que os atletas pré-mirim e mirim, em relação ao sexo os homens tem maior motivação extrínseca que as mulheres.
\end{abstract}

Palavras-chave: Treinamento. Motivação. Atletismo.

\section{Introdução}

A motivação é um fator muito importante para o ser humano na busca pelos seus objetivos. No processo de preparação e competições os treinadores consideram a motivação como fator principal, constituindo-se como elemento básico para o atleta seguir suas orientações e praticar diariamente as sessões de treinamento. (BECKER JÚNIOR, 2000).

O esporte competitivo exige dos atletas diversas adaptações em aspectos fisiológicos, biomecânicos, psicológicos e sociais. Muitos fatores influenciam diretamente o desempenho esportivo, entre eles as cargas de treinamento, as questões financeiras, a pressão dos pais, o relacionamento com o treinador, os estudos e as restrições ao lazer. Equacionar todos esses fatores exige dos atletas ótima motivação a fim de que permane- 
çam no esporte competitivo pelo maior período de tempo, objetivando realizar todas as tarefas de forma mais eficaz. (MIRANDA E BARA FILHO, 2008)

O comportamento motivacional é diferente entre os indivíduos. Conforme, Samulski (1990) classificou a motivação em dois aspectos: intrínsecos e extrínsecos. Os aspectos intrínsecos referem-se a algo interno e inseparável, que vem de dentro da pessoa; os aspectos extrínsecos estão relacionados aos fatores externos (ambientais). A razão pela qual se faz algo é apenas um meio para se alcançar outro objetivo maior, quando a ação em si só não satisfaz.

Segundo Cox (1994) a origem principal dos motivos que atraem e mantém os jovens no esporte é a motivação intrínseca. Defini-se a motivação intrínseca como a ação de uma atividade para obtenção da própria satisfação como consequência, à pessoa intrinsecamente motivada, é induzida a agir pelo prazer ou pelo desafio e não por estímulos externos, pressões ou recompensas (VALLERAND, 2007; BRICKELL E CHATZISARANTIS, 2007).

A motivação extrínseca (consciente) é caracterizada por fatores externos que podem direcionar o comportamento do atleta. Entre eles, podem-se mencionar troféus, elogios, bolsas de estudo, estrutura adequada de treinamento, um bom programa de treinamento e salários (MIRANDA E BARA FILHO, 2008). Quando a pessoa está motivada extrinsecamente, a atividade constitui apenas um meio para atingir um objetivo externo, como dinheiro ou status, esse tipo de motivação resulta em maior tensão e pressão (MASSARELLA E WINTERSTEIN, 2009).

Estudar os motivos que determinam a prática desportiva tem constituído um dos temas principais de investigação na área da Psicologia do Esporte, temos estudos em diferentes modalidades esportivas como: voleibol (FRANZONI, 2006; DA SILVA, DOS SANTOS, AGATTI, 2011), futsal (NUÑES, 2008), basquetebol (MARQUES, 2009; SALDANHA, 2008), tênis (BALBINOTTI; BALBINOTTI, 2008) e atletismo (BENTO, SILVA, PONTES, 2008).

As peculiaridades do Atletismo e o seu conjunto de provas fazem com que este esporte seja chamado de esporte de base por não apresentar grande dificuldade em sua prática, ser de fácil assimilação e por utilizar as formas básicas do movimento: andar, correr, saltar, lançar e arremessar divide-se em grupos de provas sendo: provas de pista que são todas as corridas, as de velocidade, meio-fundo, fundo e a marcha atlética, as provas de campo consistem dos saltos, lançamentos e arremesso, os saltos dividem-se em saltos horizontais: salto em distância e salto triplo e os saltos verticais: que são o salto em altura e salto com vara, já os lançamentos são dardo, disco, martelo e o arremesso do peso (NASCIMENTO, 2008).

Os praticantes do Atletismo possuem características diversificadas devido ao grande número de provas, o que exige perfis diferentes para cada uma delas. O rigor físico é fator fundamental na modalidade, sendo a potência muscular, a resistência cardiovascular e pulmonar fatores determinantes na modalidade (MORALES, et, al 2011). Essas características fazem com que o Atletismo seja um esporte pouco lúdico podendo gerar uma ausência de motivação e ocasionar o abandono da modalidade.

No campo da psicologia do esporte encontramos estudos que investigaram a motivação em atletas, do Atletismo. Podemos citar o estudo realizado com atletas pertencentes à categoria juvenil que disputaram o Campeonato Brasileiro da categoria, demonstrando que os fatores extrínsecos não são prioridades para esses atletas (DE OLIVEIRA et al., 2011), outro estudo investigou a motivação de atletas adultos do salto triplo durante o XXVII Troféu Brasil de Atletismo, encontrando a superação de si mesmo como fator motivacional mais precioso (BOMFIM, 2008) e um estudo com atletas de decatlo encontrando como principal aspecto motivador realizar um sonho, estudos 
que verificaram a motivação dos atletas durante competições (JUSTINO, 2009). Na literatura o estudo de (SILVA, 2011) pesquisou a motivação intrínseca e extrínseca em 31 atletas de rendimento do atletismo, onde só houve diferença na motivação extrínseca identificada que foi maior nos ultramaratonistas, comparados com o grupo de fundistas. Nas demais provas não ocorreram diferença na motivação, em todos os grupos a motivação intrínseca foi maior que a motivação extrínseca e não teve diferença quando comparou gênero.

Conhecer os motivos que impulsionam atletas jovens e adultos a treinarem Atletismo pode contribuir de maneira significativa, na preparação dos treinos, na captação de talentos, assim garantir uma maior aderência e permanência por parte destes em treinamentos do atletismo. Sendo assim o objetivo do presente estudo é investigar se há diferenças na motivação entre grupos de provas, categoria, sexo e nível competitivo dos atletas do atletismo.

\section{Metodologia}

\section{Amostra}

Participaram do estudo 133 atletas de Atletismo de ambos os sexos (56\% masculino) e (44\% feminino), dos núcleos de treinamento de Barueri $(n=8)$, Cria - UFJF/SESI $(n=29)$, Cria-Lavras $(n=48)$, Matias Barbosa $(n=20)$, Guarulhos-Ibirapuera $(n=14)$, Santos Dumont ( $n=6)$, Ponte Nova $(n=3)$, Usipa $(n=1)$, Caraí $(n=1)$, Gama-DF $(n=1)$, Asenpar $(n=1)$ e BMF $(n=1)$. A maioria dos atletas treinava entre 5 e 7 vezes por semana (69\%), 120 a 180 minutos por sessão (68 \%). Os atletas foram, em sua maioria, do estado de Minas Gerais, com experiência e títulos em competições de nível estadual e nacional, predominantemente. A amostra foi composta, segundo critério do atleta pela prova de sua preferência, em sua maioria por velocistas e saltadores $(58 \%)$.

\section{Instrumentos}

Para conhecimento do tempo de prática, número de sessões de treinamento por semana, especialidade do atleta, nível competitivo, foi aplicado um questionário geral. Procurando investigar a motivação utilizamos a escala de motivação esportiva (SMSSport Scale Motivation) desenvolvida por (BRIERE E PELLETIER, 1995) validada para língua portuguesa SMS-28BR (BARA FILHO et al. 2010) em estudo com atletas do voleibol, basquetebol, futsal, judô, natação, ciclismo, e tae-kwon-do, este instrumento é muito utilizado buscando identificar os níveis motivação intrínseca, extrínseca e desmotivação dos atletas nos esportes. Podemos citar os estudos, Da Costa no futebol e (LÓPES-WALLE, 2011) com boxeadores, ciclistas, esgrimistas, judocas, caratecas e nadadores. Esse índice, através das sete dimensões do SMS, determina o nível de motivação do avaliado. O questionário é composto por 28 perguntas, precedidas de um enunciado: "Por que você prática esporte?" No nosso estudo a palavra esporte foi substituída por Atletismo, onde se responde com uma escala tipo Likert de 7 pontos, que varia de "não corresponde em nada" a corresponde exatamente".

Adotou-se como critério de inclusão estar treinando há no mínimo três meses e pelo menos três vezes por semana. Todos participantes da pesquisa assinaram Termo de Consentimento Livre e Esclarecido para os atletas com idade superior aos 18 anos, e os atletas com idade inferior a 18 anos assinaram o Termo de Assentimento Livre e Esclarecido conforme normas éticas exigidas pela resolução 196/96. 


\section{Procedimentos}

A intervenção bem como a aplicação dos questionários ocorreu durante o VI Camping de Atletismo na Universidade Federal de Juiz de Fora, na Faculdade de Educação Física e Desportos durante os dias 20 a 25 de Janeiro de 2014, todos os atletas foram avisados que durante o evento estariam participando de várias pesquisas.

Para caracterização dos voluntários da amostra foram coletados os dados de massa e estatura corporal. A massa corporal foi mensurada por balança eletrônica portátil da marca Filizola, modelo ID1500, com precisão de 100 g. Todos os voluntários foram avaliados utilizando somente os respectivos shorts de treino. E a estatura corporal, foi mensurada utilizando estadiômetro portátil da marca Welmy, modelo W200/5 com precisão de $0,1 \mathrm{~cm}$. Os atletas estavam descalços.

\section{Análise Estatística}

A estatística descritiva é apresentada como média \pm desvio-padrão. Para testar diferenças no perfil motivacional entre os sexos, utilizou-se o teste $t$ de Student para amostras independentes. Em razão do tamanho amostral, optou-se pelo agrupamento de algumas categorias, descritas a seguir. Para testar diferenças entre as categorias (1-Prémirim e Mirim vs. 2-Menores vs. 3-Juvenil, Sub-23 e Adulto), entre os grupos de provas (Velocistas vs. Fundista vs. Saltadores vs. Lançadores) e entre os diferentes níveis competitivos (1-Municipal e Regional vs. 2-Estadual vs. 3-Nacional e Internacional), utilizou-se a ANOVA simples. Quando a ANOVA detectou efeitos estatisticamente significativos, procedeu-se ao teste post hoc de Tuckey. Em todas as análises, utilizou-se o software estatístico SPSS v.19 (IBM Corp.,Armonk, NY), considerando nível de significância de $5 \%(\mathrm{p} \leq 0,05)$.

\section{Resultados}

Na tabela 1 são observados os valores de média e desvio padrão, da massa corporal, estatura e idade cronológica dos voluntários da pesquisa.

Tabela 1: Características gerais da amostra, idade cronológica, massa corporal e estatura.

\begin{tabular}{lll}
\hline Variável & Sexo & Média \pm Desvio Padrão \\
Idade cronológica (anos) & Feminino & $16,0 \pm 3,7$ \\
& Masculino & $16,4 \pm 3,1$ \\
& & \\
Massa Corporal (Kg) & Feminino & $52,1 \pm 9,7$ \\
& Masculino & $62,0 \pm 15,7$ \\
Estatura $(\mathrm{cm})$ & & \\
& Feminino & $162,5 \pm 8,3$ \\
& Masculino & $171,8 \pm 10,7$
\end{tabular}


Na tabela 2 são apresentados os dados em porcentagem de categoria, nível competitivo e grupo de prova dos atletas.

Tabela 2: Categoria, nível competitivo e grupo de provas.

\begin{tabular}{lc}
\hline \multicolumn{1}{l}{ Categoria } & N\% \\
Pré-Mirim & $11(8,5 \%)$ \\
Mirim & $44(33,8 \%)$ \\
Menores & $37(28,5 \%)$ \\
Juvenil & $22(16,9 \%)$ \\
Sub-23 - Adulto & $16(12,3 \%)$
\end{tabular}

\section{Nível Competitivo}

Municipal

$11(8,4 \%)$

Regional

$11(8,4 \%)$

Estadual

$42(32,1 \%)$

Nacional

$52(39,7 \%)$

Internacional

$15(11,5 \%)$

\section{Grupo de Provas}

Velocistas

$33(25,2 \%)$

Saltadores

$43(32,8 \%)$

Fundistas

$26(19,8 \%)$

Lançadores

$26(19,1 \%)$

Combinadas

$4(3,1 \%)$

Na tabela 3, encontram-se os dados descritivos das variáveis de motivação de atletas de Atletismo de ambos os sexos e diferentes categorias. 


\section{Tabela 3: média e desvio padrão das variáveis de motivação de atletas de atletismo de ambos os sexos e diferentes categorias.}

\begin{tabular}{|c|c|c|c|c|c|c|c|c|c|c|}
\hline & & & Masculino & & & & & Feminino & & \\
\hline & $\begin{array}{l}\text { Pré- } \\
\text { Mirim }\end{array}$ & Mirim & Menores & Juvenil & $\begin{array}{l}\text { Sub-23- } \\
\text { adulto }\end{array}$ & Pré-Mirim & Mirim & Menores & Juvenil & $\begin{array}{l}\text { Sub-23- } \\
\text { adulto }\end{array}$ \\
\hline MI Saber & $5,4 \pm 1,2$ & $4,7 \pm 1,8$ & $5,0 \pm 1,5$ & $5,0 \pm 1,5$ & $5,4 \pm 1,1$ & $4,1 \pm 1,4$ & $4,2 \pm 1,5$ & $4,4 \pm 1,8$ & $5,1 \pm 1,4$ & $5,4 \pm 1,0$ \\
\hline MI conseguir & $5,0 \pm 1,1$ & $4,9 \pm 1,8$ & $4,7 \pm 1,6$ & $5,3 \pm 1,6$ & $5,0 \pm 1,1$ & $4,3 \pm 2,1$ & $4,3 \pm 1,8$ & $4,6 \pm 1,4$ & $4,9 \pm 1,4$ & $6,0 \pm 1,5$ \\
\hline MI Experiências & $4,5 \pm 1,2$ & $4,7 \pm 1,8$ & $4,9 \pm 1,3$ & $5,4 \pm 1,7$ & $5,2 \pm 1,0$ & $4,6 \pm 1,6$ & $4,1 \pm 1,6$ & $4,8 \pm 1,2$ & $5,5 \pm 1,4$ & $6,1 \pm 0,6$ \\
\hline ME Identificada & $3,8 \pm 1,3$ & $4,0 \pm 1,8$ & $4,3 \pm 1,4$ & $4,6 \pm 1,6$ & $4,3 \pm 1,5$ & $3,6 \pm 1,7$ & $3,8 \pm 1,7$ & $3,8 \pm 1,7$ & $4,8 \pm 1,0$ & $4,0 \pm 1,7$ \\
\hline $\begin{array}{l}\text { ME Introspecti- } \\
\text { va }\end{array}$ & $4,3 \pm 0,8$ & $4,1 \pm 1,7$ & $4,1 \pm 1,7$ & $4,1 \pm 2,0$ & $4,0 \pm 1,1$ & $2,4 \pm 1,4$ & $3,2 \pm 1,6$ & $3,2 \pm 1,6$ & $3,0 \pm 1,0$ & $2,7 \pm 1,5$ \\
\hline ME Externa & $4,6 \pm 1,5$ & $3,5 \pm 1,7$ & $3,6 \pm 1,6$ & $3,4 \pm 1,3$ & $3,6 \pm 1,1$ & $3,1 \pm 1,5$ & $3,0 \pm 1,3$ & $3,4 \pm 1,1$ & $3,2 \pm 1,2$ & $2,5 \pm 0,7$ \\
\hline Desmotivação & $2,3 \pm 1,2$ & $1,8 \pm 1,1$ & $1,8 \pm 0,7$ & $2,0 \pm 0,8$ & $2,1 \pm 1,0$ & $1,6 \pm 0,8$ & $1,8 \pm 1,2$ & $2,1 \pm 1,2$ & $1,5 \pm 0,6$ & $1,0 \pm 0,2$ \\
\hline $\begin{array}{l}\text { Motivação In- } \\
\text { trínseca }\end{array}$ & $\begin{array}{l}15,0 \pm \\
3,5\end{array}$ & $14,5 \pm 5,1$ & $14,7 \pm 4,0$ & $15,8 \pm 4,8$ & $15,7 \pm 3,1$ & $13,1 \pm 4,6$ & $12,7 \pm 4,5$ & $13,8 \pm 3,9$ & $15,5 \pm 3,8$ & $17,6 \pm 2,7$ \\
\hline $\begin{array}{l}\text { Motivação Ex- } \\
\text { trínseca }\end{array}$ & $\begin{array}{l}12,8 \pm \\
3,5\end{array}$ & $12,1 \pm 4,2$ & $12,1 \pm 4,2$ & $12,2 \pm 4,3$ & $12,0 \pm 2,9$ & $9,3 \pm 4,3$ & $10,0 \pm 4,1$ & $10,5 \pm 3,9$ & $11,1 \pm 2,4$ & $9,2 \pm 3,8$ \\
\hline
\end{tabular}


Na comparação entre os sexos, foi observada maior motivação extrínseca nos homens comparados às mulheres (Figura 1). Nas outras variáveis não foram observadas diferenças significativas entre os sexos $(p>0,05)$.

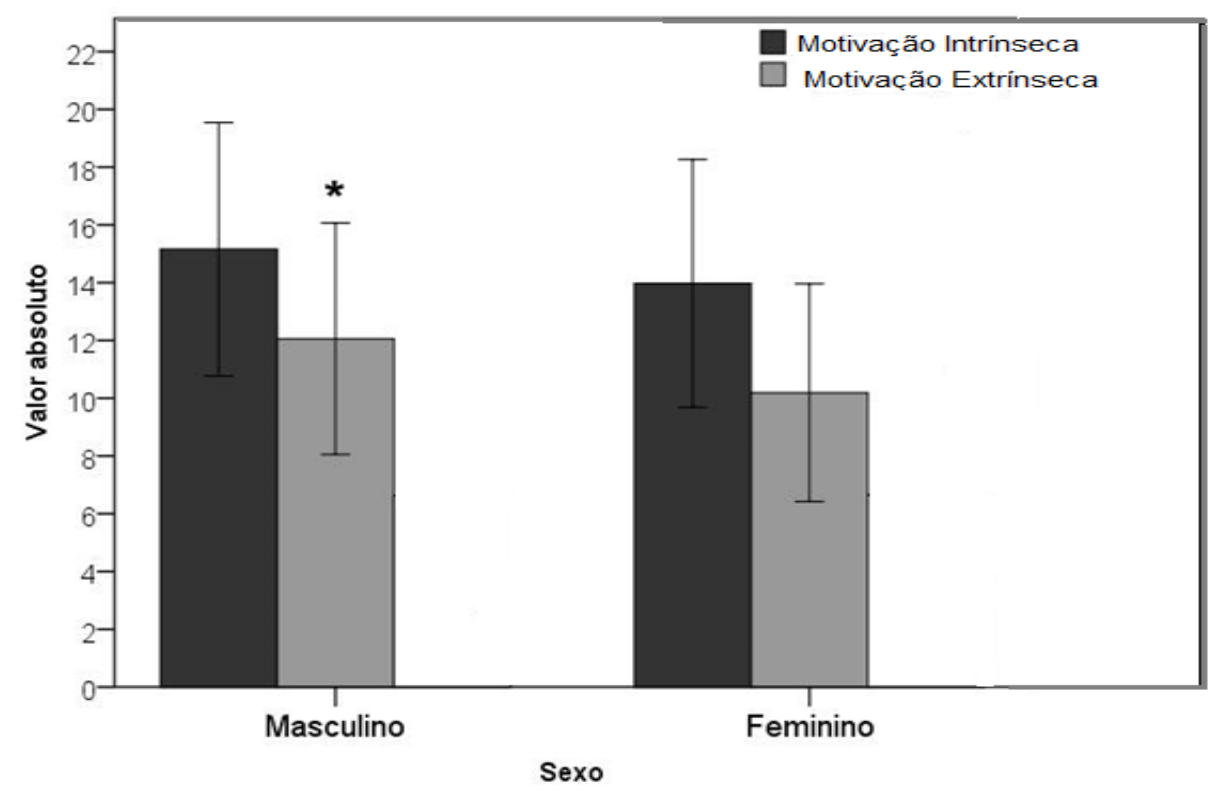

Figura 1: Média \pm desvio-padrão para os níveis de motivação extrínseca e motivação intrínseca de atletas de Atletismo do sexo masculino e feminino $(n=133)$. *diferenças significativas entre os sexos, $\mathrm{p}<0,05$.

Na Motivação Intrínseca quando comparado entre categorias, foram observados menores valores nas categorias Pré-Mirim e Mirim em relação à categoria Juvenil, Sub23 e Adultos (Figura 2). Não foram observadas diferenças significativas no perfil de motivação entre os atletas das diferentes provas do Atletismo e entre os níveis competitivos $(\mathrm{p}>0,05)$.

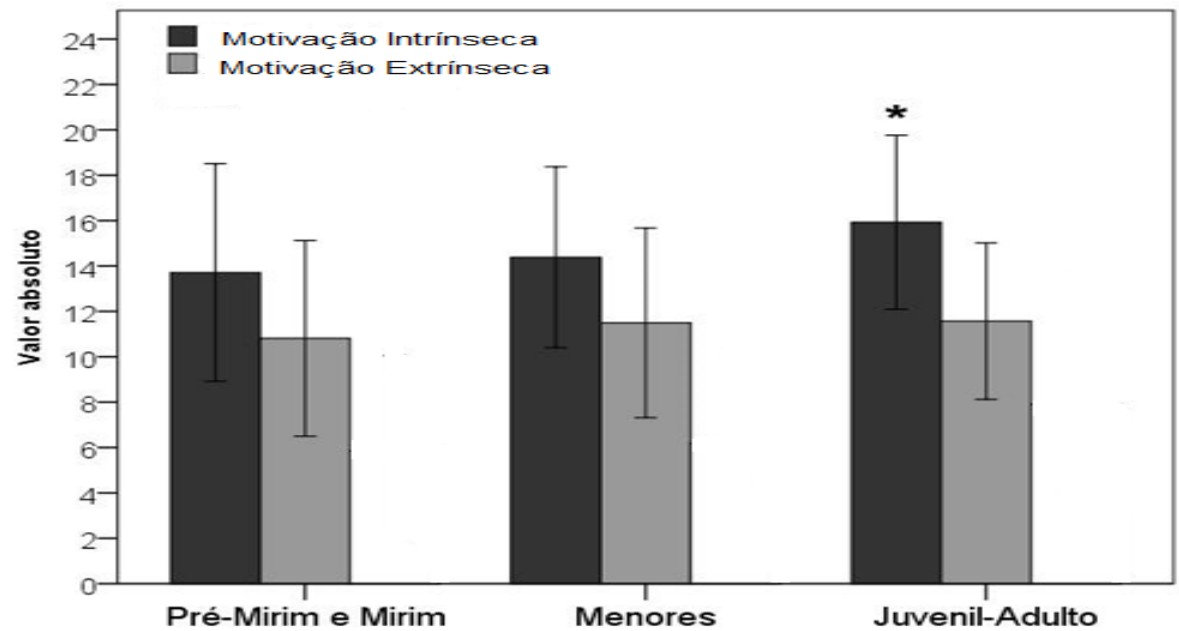

FigFigura 2: Média \pm desvio-padrão para os níveis de motivação extrínseca, motivação intrínseca de atletas de Atletismo de ambos os sexos das categorias Pré-Mirim e Mirim. $(n=55)$, Menores $(n=37)$ e Juvenil-Adulto $(n=38)$. *diferenças significativas entre Juvenil-Adulto

vs.

Pré-Mirim

e

Mirim, $\mathrm{p}<0,05$. 


\section{Discussão}

O presente estudo investigou possíveis diferenças na motivação entre grupos de provas, categoria, gênero e nível competitivo dos atletas do atletismo participantes do VI Camping de Atletismo na cidade de Juiz de Fora, Minas Gerais. A partir dos dados na comparação entre os sexos, foi observada maior motivação extrínseca nos homens comparados às mulheres. Na Motivação Intrínseca, não foram observadas diferenças significativas entre os sexos $(\mathrm{p}>0,05)$. Na comparação entre as categorias, foram observados menores valores de Motivação Intrínseca nas categorias Pré-Mirim e Mirim quando comparados à categoria Juvenil e Adultos. Não foram observadas diferenças significativas no perfil de motivação entre os atletas das diferentes provas do Atletismo e nem entre os níveis competitivos $(\mathrm{p}>0,05)$.

Entre os achados desse estudo encontramos nos homens uma maior motivação extrínseca comparado com as mulheres, nas demais variáveis não houve diferenças entre os sexos. O estudo de Bara Filho, et al., (2010) realizado com atletas de vários esportes os homens apresentaram todos os índices de motivação superiores aos das mulheres, corroborando com esses achados, Alonso et al., (2005) encontraram índices mais elevados de motivação intrínseca e na motivação extrínseca identificada em mulheres atletas, ou seja, as mulheres apresentam um componente intrínseco mais forte comparado aos homens e ainda em concordância com os achados dessa pesquisa temos os estudos com atletas gregos Doganis, (2000) e canadenses Vellerand et al., (1992).

Uma pesquisa realizada com jovens atletas de basquetebol encontrou que a motivação não sofre interferência da idade (De Oliveira et al., 2011). Entretanto no presente estudo os atletas das categorias juvenil, sub-23 e adulto apresentaram maior motivação intrínseca que os atletas das categorias pré-mirim e mirim. De acordo com esse achado e como nas categorias mais nova pré-mirim e mirim os atletas estão em fase de descoberta o estudo de Gonzales et al., (2011), mostra que atletas envolvidos em esporte competitivo são mais motivados por questões intrínsecas do que praticantes de forma recreacional, que se preocupam mais com questões ligados a motivação extrínseca como pertencer a um grupo, fato que justifica o achado do presente estudo.

O estudo com atletas de rendimento no atletismo, que contou com a participação de velocistas, meio fundistas, fundistas e ultramaratonistas mostrou que a motivação dos corredores de diferentes especialidades foi similar, com exceção da sub-escala de motivação extrínseca identificada, que evidenciou diferença estatisticamente significativa entre os grupos corredores meio fundistas e ultramaratonistas, superior no segundo grupo (SILVA, 2013). Ainda que com algumas diferenças nas variáveis analisadas constatássemos que ambas as motivações estão presentes no cotidiano de treinamentos tanto de jovens como de adultos e contribuindo com estes achados os estudos de Gonzales; Valle; Marques, (2011); Martin; Coe, (2001); Miranda; Bara Filho, (2008) apontam que a motivação intrínseca e a extrínseca são muito importantes tanto nos treinamentos e nas competições. Contudo Mallet e Hanrahan (2004); Krouse et. al (2011); Zaar e Reis (2010) e Zaar e Balbinotti (2011), encontraram a motivação intrínseca como maior predominância comparada com a extrínseca.

Os achados deste estudo são de extrema importância para que o conteúdo intrínseco seja bastante trabalhado, principalmente nos homens, pois, estes mostraram maior motivação extrínseca. Através desse estudo treinadores e profissionais do Atletismo e dos demais esportes, devem olhar com mais cuidado para as questões motivacionais dos seus atletas, criarem estratégias a partir destes achados para que se consigam cada vez mais adeptos ao atletismo, e que estes atletas estejam sempre focados e motivados intrinsecamente, uma vez 
que os componentes extrínsecos quando não bem trabalhados, pode levar o atleta a se manter na modalidade apenas pelas recompensas. A literatura apresenta trabalhos com essa magnitude de investigação, em outras modalidades esportivas, porém no atletismo ainda são poucos os estudos que fez esse tipo de investigação, comparando a motivação entre categorias, grupos de provas e sexo, faz-se necessário que haja mais estudos com essa temática, com um maior número de indivíduos, que as categorias tenham grupos mais homogêneos, tanto com a faixa etária e também por provas do atletismo, que se utilize de outros métodos de investigação que não só o questionário, para contribuir com os achados dessa e de futuras pesquisas.

\title{
Conclusão
}

A motivação para a prática do atletismo não depende da prova que se disputa. Atletas das categorias juvenis, sub-23 e adultos são mais motivados intrinsecamente que os prémirim e mirim, e os homens apresentaram uma maior motivação extrínseca do que as mulheres.

\section{MOTIVATION OF YOUNG PEOPLE AND ADULTS FOR ATHLETICS TRAIN- ING}

\begin{abstract}
The study investigated differences on the motivation of Athletics athletes, comparing tests groups, competitive level, category and gender. We evaluated the motivation through the questionnaire (SMS - BR28) of 133 athletes, 56\% male and 44\% female. There were no significative differences between tests groups and competitive level, significance level $(\mathrm{p}<0,05)$. There was significant difference comparing the young and adults motivation to the under-child and child. We concluded that the motivation to train Athletics independs on the disputed test, young athletes, under- 23 and adults are more motivated intrinsically that the under-child and child athletes, related to the gender, men have bigger extrinsic motivation tham women.
\end{abstract}

Keywords: Training. Motivation. Athletics.

\section{MOTIVACIÓN DE LOS JÓVENES Y ADULTOS PARA EL ENTRENA- MIENTO DE ATLETISMO}

\section{Resumen}

El estúdio investigó las diferencias em la motivación de los atletas de Atletismo, em comparación con grupos de pruebas, nivel competitivo, categoría e género. Evaluamos la motivación através del cuestionario (SMS - BR28) de 133 atletas, 56\% masculino y 44\% femenino. No hubo diferencias significativas entre grupos de pruebas y nivel competitivo, nivel de significación $(\mathrm{p}<0,05)$. Había diferencia significativa em comparación com la motivación del juveniles y adultos contra pre-junior y junior. Concluimos que la motivación para entrenar Atletismo es independiente de las pruebas disputadas, atletas juveniles, sub-23 y adultos son más motivados intrínsecamente que los atletas pre-junior y junior, enrelación al género los hombres tiene una mayor motivación extrínseca que lãs mujeres.

|

Palabras clave: Entrenamiento.Motivación. Atletismo. 


\section{Referências}

ALONSO, J. L. N.; LUCAS, J. M.; IZQUIERDO, J. G. N. Validacion de la version espanola de la Echelle de Motivation en Education. Psicothema, v.17, n.2, p.344-349, 2005.

BALBINOTTI, M. A.A.; BALBINOTTI, C. A.A. Motivação e Perspectiva Futura no Tênis Infanto-juvenil. Revista Brasileira de Psicologia do Esporte, v. 2, n. 2, p. 1-20, 2008. ISSN 1981-9145

BARA FILHO, M.; ANDRADE, D.; MIRANDA. R.; NÚNEZ, L.J.; MARTÍN-ALBO, J.; RIBAS, P.R.; . Preliminary Validation of Brazilian Version of the Sport Motivation Scale. UniversitasPsychologica, v. 10, n. 2, p. 557-566, 2010. ISSN 1657-9267

BECKER JR., B. Manual de psicologia do esporte e exercício. Porto Alegre: Nova Prata, 2000.

BENTO, A. N. F.; SILVA, M. R.; PONTES, M. Motivação de Atletas Federados para a Prática de Atletismo. 2008.

BOMFIM, A. Fatores Motivacionais dos Triplistas Participantes do XXVII Troféu Brasil de Atletismo. 2008.

BRICKELL, T.A.; CHATZISARANTIS, N.L.D.. Using self-determination theory to examine the motivation correlates and predictive utility of spontaneous exercise implementation intentions. Psychology of Sport and Exercise, vol. 8, p. 758-770, 2007.

BRIERE, N.; VALLERAND, R.; BLAIS, N.; PELLETIER, L. Developpement et validation d'unemesure de motivation intrinseque, extrinseque et d'amotivation en contextesportif : 1 'Echelle de motivation dans les sports (EMS). International Journal of Sport Psychology, v.26, p.465-489, 1995.

COX, R.H. Sport psychology: concepts and applications. 3rd ed. Dubuque: Brown \& Benchmark, 1994.

DA COSTA, V. T.; ALBUQUERQUE, M. R.; LOPES, M. C.; NOCE, F.; DA COSTA, I.T.; FERRREIRA, R. M.; SAMULSKI, D. M. Validação da Escala de Motivação no Esporte (SMS) no Futebol para Língua Portuguesa Brasileira. Revista Brasileira de Educação Física e Esporte, v. 25, n. 3, p. 537-546, 2011. ISSN 1981-4690.

DECI, E. L., RYAN, R. M. Intrinsic motivation and self-determination in human behavior, New York: Plenum Press. 1985.

DOGANIS, G. Development of a Greek version of the Sport Motivation Scale. Perceptual and Motor Skills, v.90, p.505-512, 2000.

FRANZONI, T. D. B. Fatores Motivacionais Associados à Prática Esportiva de Atletas de Voleibol. 2006. 
GONZALEZ, O. M.; VALLE A. S.; MARQUEZ, S. Autodeterminacion y adherencia al ejercicio: estado de la cuestion Self-determination and exercise adherence: state-ofthe- art. International Journal of Sport Science, v.3, p.287-304, 2011.

JUSTINO, P. E. D. S. Caracterização dos Fatores Motivacionais dos Decatletas Participantes da III Copa São Paulo de Provas Combinadas. 2009.

KROUSE, R.Z; RANSDELL, L.B.; LUCAS, S.M.; PRITCHARD, M.E. Motivation, goal orientation, coaching, and training habits of women ultrarunners. Journal Strength Conditional Research, 25(X): 000-000, 2011.

LÍVIA TAVARES DA SILVA, C.; PATRÍCIA DOS SANTOS, V.; SÍlVIA MARIA AGATTI, L. Fatores Motivacionais de Jovens Atletas de Vôlei. Revista Brasileira de Ciências do Esporte, v. 33, n. 2, p. 303, 2011. ISSN 01013289.

LÓPEZ-WALLE, J.; BALAGUER. I.; CASTILHO. I.; TRISTÁN. J. Clima Motivacional Percibido, Motivación Autodeterminada y Autoestima em Jóvenes Deportistas Mexicanos. RevPsicol Deporte, v. 20, n.1, p. 209-22, 2011.

MALLET, C.; HANRAHAN, S. J. Elite athletes: why does the fire bum so brightly? Psychology of Sport and Exercise, v.5, p.182-200, 2004.

MARQUES, S. C. Competências Mentais, Ansiedade Competitiva e Motivação em Atletas de Basquetebol. Ferreira, J. P. L. e Gaspar, P. M. P. 2009.

MASSARELLA, Fábio Luiz; WINTERSTEIN, Pedro José. Motivação intrínseca e o estado mental Flow em corredores de rua. Movimento (ESEF/UFRGS), v. 15, n. 2, p. 45-68, 2009.

MARTIN, D. E.; COE, P. N. Entrenamiento para corredores de fondo y médio fondo. 3a ed. Editorial Paidotribo, 2001.

MICHELE DONATO DE OLIVEIRA, E. G. A., WELTON GUSTAVO DE SOUZA PINTOR, PABLO JORDÃO, ADRYA ESTEVÃO VENÂNCIO, RAFAEL OCTAVIANO DE SOUZA, SÉRGIO ROBERTO ADRIANO PRATI. Estado de Motivação e IMC em Atletas do Campeonato Brasileiro Juvenil de Atletismo. 2011.

MIRANDA, R.; BARA FILHO, M. Construindo um atleta vencedor: uma a abordagem psicofísica do esporte. Grupo A, 2008. ISBN 8536314257

MORALES, Anderson Pontes et al. Influência do Gênero nos Testes de vo2máx e Rast em Atletas de Atletismo. Biológicas \& Saúde, v. 1, n. 2, 2011.

NASCIMENTO, A. Pedagogia do esporte e o atletismo: considerações da iniciação e da especialização esportiva precoce. Campinas-SP: (Dissertação Mestrado) FEF/UNICAMP, v. 6, n. 1, 2008.

NUÑEZ, P. R. M.; DE LARA PICADA, H.F.S.; SCHULZ, S.T.; HABITANTE, C. A.; DA SILVA, J.V.P. Motivos que levam adolescentes a praticarem futsal. Conexões: Revista da Faculdade de Educação Física da UNICAMP, v.6, n.1, 2008. 
OLIVEIRA, H. Z. D. Motivação e Flow-Felling em Jovens Jogadores de Basquetebol, 2013. Dissertação apresentada ao Programa de Pós-graduação em Educação Física are de concentração: Movimento Humano, da Universidade Federal de Juiz de Fora, como requisito para obtenção do grau de Mestre. (dados não publicados)

PELLETIER, L. G.; FORTIER, M. S.; VALLERAND, R. J.; TUSON, K. M.; BRIERE, N. M.; BLAIS, M. R. Toward a new measure of intrinsic motivation, extrinsic motivation, and amotivation in sports: The Sport Motivation Scale (SMS).Journal of Sport \& Exercise Psychology, n.17, p.35-53, 1995.

SALDANHA, R. P. Motivação à prática regular de atividades físicas? Um estudo com atletas de basquetebol infanto-juvenis (13 a 16 anos). 2008.

SILVA, M. V. D. Estado Mental Flow e motivação no atletismo: dos velocistas aos ultramaratonistas. 2013. Dissertação apresentada ao Programa de Pós-graduação em Educação Física are de concentração: Movimento Humano, da Universidade Federal de Juiz de Fora, como requisito para obtenção do grau de Mestre. (dados não publicados)

SAMULSKI, D. M. Psicologia do esporte. Belo Horizonte: Ed. UFMG, 1990.

VALLERAND, R. J.. A hierarchical model of intrinsic and extrinsic motivation for Sport and physical activity. In: HAGGER, M.S.; CHATZISARANTIS, N.L.D. (eds.). Intrinsic Motivation and self-determination in exercise and sport. Human Kinetics, 2007. P.255280

VALLERAND, R. J.; PELLETIER, L. G.; BLAIS, M. R.; BRIERE, N. M.; SENECAL, C.; VALLIERES, E. F. The Academic Motivation Scale: a measure of intrinsic, extrinsic and amotivation in education. Educational and Psychological Measurement, v.52, p.1003-1017, 1992.

ZAAR, A.; BALBINOTTI, M. A. A. A descriptive study of the dimensions of motivation Runners 5.000 and 10.000 metros Brazil Trophy of Athletics. EFDeportes.com, Revista Digital. Buenos Aires, v.152, 2011.

ZAAR, A.; REIS, V. Um Estudo Descritivo das Dimensoes Motivacionais de Atletas Corredores de Meio-Fundo. Simposio Internacional Promocao da Saude e Actividade Fisica: Contributos para o Desenvolvimento Humano. Livro Promocao da Saude Universidade de Tras-os-Montes e Alto Douro - Centro de Investigacao em Desporto, Saude e Desenvolvimento Humano 1.a Ed.: Julho de 2010.

Recebido em: 01/04/2016

Revisado em: 11/07/2016

Aprovado em: 01/09/2016

Endereço para correspondência:

renatosiqueiraedufisica@gmail.com

Renato Siqueira de Souza

Universidade Federal de Juiz de Fora

Rua José Lourenço Kelmer, S/n - Martelos

Juiz de Fora - MG, 36036-330 